\title{
Protein C-ets-2
}

National Cancer Institute

\section{Source}

National Cancer Institute. Protein C-ets-2. NCI Thesaurus. Code C17482.

Protein C-ets-1 (441 aa, $~ 50 \mathrm{kDa}$ ) is encoded by the human ETS1 gene. This protein plays a role in transcriptional regulation that is associated with cell motility, apoptosis induction, cell cycle regulation and immune responses. 